PROCEEDINGS OF THE

AMERICAN MATHEMATICAL SOCIETY

Volume 132, Number 3, Pages 827-830

S 0002-9939(03)07125-9

Article electronically published on July 28, 2003

\title{
ENCLOSURE RESULTS \\ FOR SECOND-ORDER RELATIVE SPECTRA BY ELEMENTARY MEANS
}

\author{
PETER OTTE
}

(Communicated by Joseph A. Ball)

\begin{abstract}
Motivated by the general approach due to Shargorodsky we derive enclosure results for the second-order relative spectrum of bounded selfadjoint operators by studying quadratic operator pencils. The quality of the results is discussed by means of a simple example.
\end{abstract}

Higher-order relative spectra of selfadjoint operators were introduced by Davies [1], who used, particularly, second-order spectra as a new definition for resonances (see Howland [2]). Subsequently, Shargorodsky [5] obtained general enclosure results for the $n$-th order spectrum.

Here we focus on second-order spectra whereby completely elementary tools from Hilbert space theory suffice not only to rediscover but also to strengthen some of the enclosure results. The key idea is to write the operator being considered as an operator block matrix and thus to link the question of second-order spectra with the invertibility of some special quadratic operator pencils.

Let $\mathcal{H}$ be a complex Hilbert space, $A, B: \mathcal{H} \rightarrow \mathcal{H}$ bounded selfadjoint operators, and $\mathbb{1}: \mathcal{H} \rightarrow \mathcal{H}$ the identity. We consider the quadratic operator pencil

$$
M(z):=(A-z \mathbb{1})^{2}+B^{2}, z \in \mathbb{C},
$$

and define

$$
\rho(A, B):=\{z \in \mathbb{C} \mid M(z) \text { has a bounded inverse }\}
$$

and $\sigma(A, B):=\mathbb{C} \backslash \rho(A, B)$. These resemble the ordinary resolvent set and spectrum. We introduce

$$
\begin{array}{rlrl}
\alpha_{\text {min }} & :=\inf \sigma(A) & \beta_{\text {min }}:=\left(\inf \sigma\left(B^{2}\right)\right)^{1 / 2} & \gamma_{\text {min }}:=\left(\inf \sigma\left(A^{2}+B^{2}\right)\right)^{1 / 2} \\
\alpha_{\text {max }}:=\sup \sigma(A) & \beta_{\text {max }}:=\left(\sup \sigma\left(B^{2}\right)\right)^{1 / 2} & \gamma_{\max }:=\left(\sup \sigma\left(A^{2}+B^{2}\right)\right)^{1 / 2}
\end{array}
$$

with $\sigma$ denoting the (usual) spectrum of the respective operator.

Theorem 1. Let $z \in \sigma(A, B)$ with $z=x+i y, x, y \in \mathbb{R}$. Then:

(i) $x \in\left[\alpha_{\min }, \alpha_{\max }\right]$. If $y=0$, then $x \in \sigma(A) \subset\left[\alpha_{\min }, \alpha_{\max }\right]$.

(ii) $|y| \in\left[\beta_{\min }, \beta_{\max }\right]$.

(iii) $\gamma_{\min } \leq|z| \leq \gamma_{\max }$.

Received by the editors October 17, 2002 and, in revised form, November 1, 2002.

2000 Mathematics Subject Classification. Primary 47A10, 47B15.

I would like to thank H. Kalf for encouraging me to prepare this note. 
Proof. To show invertibility of $M(z)$ we invoke the Lax-Milgram Theorem (see, e.g., [4]) if not indicated otherwise.

i) Using the triangle inequality we obtain

$|\operatorname{Im}(\varphi, M(z) \varphi)| \geq 2|y|\left(x-\alpha_{\max }\right)\|\varphi\|^{2}$ and $|\operatorname{Im}(\varphi, M(z) \varphi)| \geq 2|y|\left(\alpha_{\min }-x\right)\|\varphi\|^{2}$, which proves the case $y \neq 0$ and $x \notin\left[\alpha_{\min }, \alpha_{\max }\right]$.

If $y=0$ and $x \notin \sigma(A)$ the statement is implied by

$$
\operatorname{Re}(\varphi, M(z) \varphi)=\|(A-x \mathbb{1}) \varphi\|^{2}+\left(\varphi, B^{2} \varphi\right) \geq \frac{1}{\left\|(A-x \mathbb{1})^{-1}\right\|^{2}}\|\varphi\|^{2} .
$$

ii) The left boundary follows from

$$
\operatorname{Re}(\varphi, M(z) \varphi)=\|(A-x \mathbb{1}) \varphi\|^{2}+\left(\varphi, B^{2} \varphi\right)-y^{2}\|\varphi\|^{2} \geq\left(\beta_{\text {min }}^{2}-y^{2}\right)\|\varphi\|^{2} .
$$

If $|y|>\beta_{\max }$, we write

$$
M(z)=(A-z \mathbb{1})^{2}\left[\mathbb{1}+\left((A-z \mathbb{1})^{-1}\right)^{2} B^{2}\right] .
$$

The statement is then proved with the aid of the norm estimate for the resolvent (see, e.g., 3]), which yields

$$
\left\|\left((A-z \mathbb{1})^{-1}\right)^{2} B^{2}\right\| \leq \frac{\beta_{\max }^{2}}{y^{2}} .
$$

iii) If $y=0$, then $x \in \sigma(A)$ by i) and hence $|x| \leq \max \left\{\left|\alpha_{\min }\right|,\left|\alpha_{\max }\right|\right\} \leq \gamma_{\max }$.

Let $y \neq 0$. Then we may eliminate $(\varphi, A \varphi)$ from the real part by dint of the imaginary part and, having rearranged and taken absolute values, we obtain

$$
\left|\left(x^{2}+y^{2}\right)\|\varphi\|^{2}-\left(\varphi,\left(A^{2}+B^{2}\right) \varphi\right)\right|=\left|-\operatorname{Re}(\varphi, M(z) \varphi)+\frac{x}{y} \operatorname{Im}(\varphi, M(z) \varphi)\right| .
$$

Applying the Cauchy-Schwarz inequality to the right-hand side and estimating the left-hand side from below yield

$$
C\|\varphi\|^{2} \leq\left(1+\frac{x^{2}}{y^{2}}\right)^{1 / 2}|(\varphi, M(z) \varphi)|
$$

with either $C=x^{2}+y^{2}-\gamma_{\max }^{2}$ or $C=\gamma_{\min }^{2}-x^{2}-y^{2}$, which proves the statement.

We discuss the above bounds. For $\alpha:=\max \left\{\left|\alpha_{\min }\right|,\left|\alpha_{\max }\right|\right\}$ we have $0 \leq \alpha \leq$ $\gamma_{\max }$. Furthermore, $0 \leq \beta_{\max } \leq \gamma_{\max }$. Note that $\beta_{\min } \leq \beta_{\max }$ because $B^{2} \geq 0$. In the case $\alpha^{2}+\beta_{\max }^{2} \leq \gamma_{\max }^{2}$ combining bounds i) and ii) gives a sharper result than iii). If $\alpha^{2}+\beta_{\max }^{2}>\gamma_{\max }^{2}$, then all three bounds i) through iii) must be used in order to yield the best estimates. The interested reader may draw some pictures by himself to visualize these results.

Thus far we have disregarded the relationship between $A$ and $B$, e.g., commutation properties. One could hope for more accurate estimates by using such properties. We leave this for future investigations.

For a bounded selfadjoint operator $H: \mathcal{H} \rightarrow \mathcal{H}$ and an orthogonal projection $P: \mathcal{H} \rightarrow \mathcal{H}$ Davies [1] defines the $n$-th order relative resolvent set and spectrum of $H$ by

$$
\rho_{n}(H, P):=\left\{z \in \mathbb{C} \mid P(H-z \mathbb{1})^{n} P: \operatorname{ran} P \rightarrow \operatorname{ran} P \text { has a bounded inverse }\right\}
$$


and $\sigma_{n}(H, P):=\mathbb{C} \backslash \rho_{n}(H, P)$, respectively. We write $\mathcal{H}$ as an orthogonal sum $\mathcal{H}=\mathcal{H}_{1} \oplus \mathcal{H}_{2}$ with $\mathcal{H}_{1}:=\operatorname{ran} P$ and $\mathcal{H}_{2}:=\mathcal{H}_{1}^{\perp}$ and $H$ as a $2 \times 2$ block matrix:

$$
H=\left(\begin{array}{ll}
H_{11} & H_{12} \\
H_{21} & H_{22}
\end{array}\right), H_{j k}: \mathcal{H}_{k} \rightarrow \mathcal{H}_{j}, j, k=1,2 .
$$

It is easily seen that the $H_{j k}$ are bounded and obey $H_{j k}^{*}=H_{k j}$. In the case $n=2$,

$$
M(z):=P(H-z \mathbb{1})^{2} P=\left(H_{11}-z \mathbb{1}\right)^{2}+H_{12} H_{21}: \mathcal{H}_{1} \rightarrow \mathcal{H}_{1} .
$$

This is a quadratic operator pencil with $H_{12} H_{21} \geq 0$, wherefore we can apply Theorem 1 by putting $A:=H_{11}$ and $B^{2}:=H_{12} H_{21}$. We do this for iii). The accordant spectral quantity, $\gamma_{\max }$, can be estimated further:

$$
\gamma_{\text {max }}^{2}=\sup \sigma\left(H_{11}^{2}+H_{12} H_{21}\right)=\sup _{P \psi \neq 0} \frac{\left(P \psi, H^{2} P \psi\right)}{\|P \psi\|^{2}} \leq\|H\|^{2} .
$$

We then deduce immediately

Theorem 2. Let $H: \mathcal{H} \rightarrow \mathcal{H}$ be a bounded selfadjoint operator. Then,

$$
\sigma_{2}(H, P) \subset\left\{z \in \mathbb{C}|| z \mid \leq \gamma_{\max }\right\} \subset\{z \in \mathbb{C}|| z \mid \leq\|H\|\} .
$$

As we show simple examples of $3 \times 3$ diagonal matrices, in general, the strict inequality $\gamma_{\max }<\|H\|$ holds; whence the first inclusion in Theorem 2 provides more accurate information on $\sigma_{2}(H, P)$ than the second one, which is a special case of a theorem of Shargorodsky 5 .

The larger circle in Theorem 2 is optimal if the spectrum of $H$ is symmetric with respect to the real axis, which we can always achieve by shifting the spectrum.

Corollary 3. Let $H: \mathcal{H} \rightarrow \mathcal{H}$ be bounded and selfadjoint and

$$
\delta_{\min }:=\inf \sigma(H), \delta_{\max }:=\sup \sigma(H) .
$$

Then,

$$
\sigma_{2}(H, P) \subset\left\{z \in \mathbb{C}|| z-\frac{1}{2}\left(\delta_{\min }+\delta_{\max }\right) \mid \leq \frac{1}{2}\left(\delta_{\max }-\delta_{\min }\right)\right\} .
$$

Proof. Apply Theorem 2 to the operator $\tilde{H}:=H-\frac{1}{2}\left(\delta_{\min }+\delta_{\max }\right) \mathbb{1}$.

Corollary $[3$ is best possible under the assumptions given in that the radius of that circle cannot be lessened and a circle is the only possible curve that can enter in any theorem of this type. To see this consider

$$
H(\lambda, V):=\left(\begin{array}{cc}
\lambda & V \\
\bar{V} & -\lambda
\end{array}\right), \lambda \in \mathbb{R}, V \in \mathbb{C}, \quad P:=\left(\begin{array}{ll}
1 & 0 \\
0 & 0
\end{array}\right) .
$$

$H(\lambda, V)$ has eigenvalues $\pm \sqrt{\lambda^{2}+|V|^{2}}$ and, consequently, $\|H(\lambda, V)\|=\sqrt{\lambda^{2}+|V|^{2}}$. Finally, $M(z)=(\lambda-z)^{2}+|V|^{2}$, which is invertible if and only if $M(z) \neq 0$, whence

$$
\sigma_{2}(H(\lambda, V), P)=\{\lambda \pm i|V|\} .
$$

If $z \in \sigma_{2}(H(\lambda, V), P)$ we have $|z|=\sqrt{\lambda^{2}+|V|^{2}}=\|H\|$ and $x=\lambda$. For $\|H\|$ fixed and $V:=\sqrt{\|H\|^{2}-\lambda^{2}}$ the circle in Corollary[3 is the same for all $\lambda \in[-\|H\|,\|H\|]$ and $\sigma_{2}(H(\lambda, V), P)$ runs through the whole circle while $\lambda$ runs from $-\|H\|$ to $\|H\|$. 


\section{REFERENCES}

1. E. B. Davies, Spectral enclosures and complex resonances for general self-adjoint operators, LMS J. Comput. Math. 1 (1998), 42-74. MR 2000e:47043

2. J. S. Howland, The Livsic matrix in perturbation theory, J. Math. Anal. Appl. 50 (1975), 415-437. MR 51:11153

3. T. Kato, Perturbation theory for linear operators, Second edition, Grundlehren der Mathematischen Wissenschaften, Band 132, Springer-Verlag, Berlin-New York, 1976. MR 53:11389

4. R. Kress, Linear integral equations, Second edition, Applied Mathematical Sciences 82, Springer-Verlag, New York, 1999. MR 2000h:45001

5. E. Shargorodsky, Geometry of higher order relative spectra and projection methods, J. Operator Theory 44 (2000), no. 1, 43-62. MR 2001f:47004

Mathematisches Institut, Universität München, Theresienstrasse 39, 80333 München, GERMANY

E-mail address: otte@mathematik.uni-muenchen.de

Current address: Fakultät für Mathematik, Ruhr-Universität Bochum, Universitätsstrasse 150, 44780 Bochum, Germany

E-mail address: Peter.Otte@ruhr-uni-bochum.de 